\title{
THURSTON NORM AND TAUT BRANCHED SURFACES
}

\author{
B. D. STERBA-BOATWRIGHT
}

(Communicated by Haynes R. Miller)

\begin{abstract}
Let $x$ denote the Thurston norm on $H_{2}(N ; \mathbf{R})$, where $N$ is a closed, oriented, irreducible, atoroidal three-manifold $N$. U. Oertel defined a taut oriented branched surface to be a branched surface with the property that each surface it carries is incompressible and $x$-minimizing for the (nontrivial) homology class it represents. Given $\varphi$, a face of the $x$-unit sphere in $H_{2}(N ; \mathbf{R})$, Oertel then asks: is there a taut oriented branched surface carrying surfaces representing every integral homology class projecting to $\varphi$ ? In this article, an example is constructed for which the answer is negative.
\end{abstract}

0. Introduction. Let $x$ denote the Thurston norm on $H_{2}(N ; \mathbf{R})$ for a closed, oriented, irreducible, atoroidal three-manifold $N$. Following the definition of U. Oertel, let a taut oriented branched surface be a branched surface $\mathbf{B}$ with the property that each surface carried by $\mathbf{B}$ is incompressible and $x$-minimizing for the (nontrivial) homology class it represents. If $\varphi$ is a face of the $x$-unit sphere in $H_{2}(N ; \mathbf{R})$, we say that $\varphi$ is spanned by a branched surface $\mathbf{B}$ if $\mathbf{B}$ carries surfaces representing every integral homology class which projects to $\varphi$. Let $V_{1}, \ldots, V_{k}$ be the vertices of $\varphi$, and let $\mathbf{B}$ be a taut oriented branched surface spanning $\varphi$. Then $\mathbf{B}$ carries surfaces $F_{1}, \ldots, F_{k}$ representing integral classes which project to $V_{1}, \ldots, V_{k}$. Further, if + denotes oriented cut-and-paste, then $\mathbf{B}$ must also carry $F_{1}+\cdots+F_{k}$, which by definition is then incompressible and $x$-minimizing. In this note, we present an example of $N$ and $\varphi$ with the property that any $x$-minimizing surfaces representing classes projecting to certain vertices of $\varphi$ have a compressible cut-and-paste sum. Thus, no branched surface spanning $\varphi$ could be a taut oriented branched surface.

The construction of $N$ is in two parts. In $\S 1$, we construct a manifold with boundary $M$ which in fact would serve as $N$ if it were closed. In $\S 2$, we rectify this by gluing two copies of $M$ together to get $N$ without changing the relevant properties of $M$.

1. Construction of $M$. Let $f_{i}(i=1,2,3)$ be copies of a twice punctured torus. Attach $f_{i} \times I(i=1,2,3)$ to a solid torus $V$, gluing $\left(\partial f_{i}\right) \times I$ along longitudinal annuli of $\partial V$ according to the schematic in Figure $1(I=[0,1])$. Call the result $M$. Let $F_{i}$ be the genus-2 surface formed from $f_{i} \times \frac{1}{2}$ by attaching an essential annulus in $V$ to the boundary components of $f_{i} \times \frac{1}{2}$ (see $F_{1}$ in Figure 1). $M$ has two boundary components, $\partial_{0} M$ and $\partial_{1} M$, where $\left(f_{i} \times j\right) \subset \partial_{j} M$. Orient $M, F_{i}$ so

Received by the editors December 15, 1986.

1980 Mathematics Subject Classification (1985 Revision). Primary 57N10; Secondary 57M99.

Key words and phrases. Branched surface, taut oriented branched surface, Thurston norm, $x$ minimizing surface. 


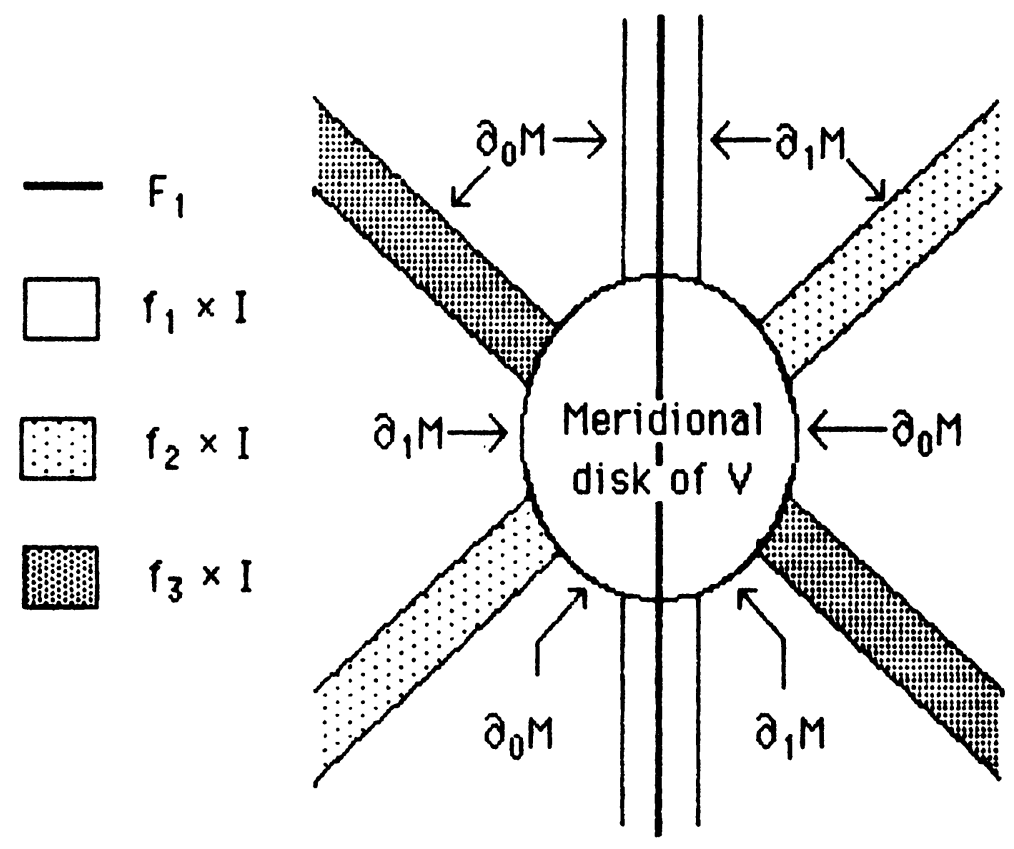

FIGURE 1

that the transverse direction to $f_{i} \times \frac{1}{2}$ runs from $f_{i} \times 0$ to $f_{i} \times 1$. Consider $\partial V$ as the union of two sets of six longitudinal annuli apiece, $\omega=\bigcup\left(\partial f_{i}\right) \times I$ and $\rho=\overline{\partial V \backslash \omega}$.

LEMMA 1. (i) $M$ is irreducible and atoroidal.

(ii) $\partial_{0} M$ and $\partial_{1} M$ are $x$-minimizing in $H_{2}(M)$.

(iii) If $H_{i}$ is a genus-2 surface in $M$ with $\left[H_{i}\right]=\left[F_{i}\right] \in H_{2}(M)$, then $H_{i}$ is isotopic to $F_{i}$.

(iv) If $H_{i}(i=1,2,3)$ is as above, $H_{1}+H_{2}+H_{3}$ contains a closed component entirely in $V$, and is therefore compressible.

PROOF. (i) is obvious.

(ii) Any $\chi_{-}$-minimizing surface $G$ homologous to (say) $\partial_{0} M$ must intersect $V$ in annuli and $f_{i} \times I$ in a surface with Euler characteristic -2 . Therefore, $\chi_{-}(G) \geq$ $6=\chi_{-}\left(\partial_{0} M\right)$.

(iii) $H_{i} \cap\left(f_{j} \times I\right)(i \neq j)$ must consist of annuli parallel to $\left(\partial f_{j}\right) \times I$. These can be removed by isotopy. Similarly, $H_{i} \cap\left(\left(\partial f_{i}\right) \times I\right)$ may be made a single simple closed curve.

(iv) The isotopy in (iii) may be performed on $\omega$ rather than on $H_{i}$. Thus, assume that $C_{i}=V \cap H_{i}$ is an annulus and that $\left(\cap H_{i}\right)=\left(\cap C_{i}\right) \subset V$. If $C_{1}+C_{2}$ contains a closed component $W$, then either $C_{3} \cap W=\varnothing$ or $C_{3}$ separates $W$. In the latter case, one "half" or the other gives rise to a closed component of $C_{1}+C_{2}+C_{3}$.

Assume, therefore, that $C_{1}+C_{2}$ is two annuli $D_{+}$and $D_{-}$, with $D_{+}$(resp., $D_{-}$) cutting out a solid torus $Y_{+}$(resp., $\left.Y_{-}\right)$from $V$ with the transverse direction pointing out of $Y_{+}$(resp., into $Y_{-}$). Now $C_{1} \cup C_{2}$ separates the two components of $\partial C_{3}$, so $C_{3} \cap\left(C_{1}+C_{2}\right)$ is not empty: say that $C_{3} \cap D_{+} \neq \varnothing$. Let the subtorus of 
$V \backslash C_{3}$ with the transverse direction of $C_{3}$ pointing out be called $X$. Then $Y_{+} \cap X \neq$ $\varnothing$ and $Y_{+} \cap X \cap \partial D_{+}=\varnothing$, so $D_{+}+C_{3}$ has a closed component. Q.E.D.

The following lemma will be needed in the next section.

LEMMA 2. Let $\gamma_{0}$ be a simple closed curve in $\partial_{0} M$ such that $\left[\gamma_{0}\right] \neq 1 \in$ $\pi_{1}\left(\partial_{0} M\right)$ and one of the following holds:

(i) $\exists$ a simple closed curve $\gamma_{1} \subset \partial_{1} M$ such that $\gamma_{0} \cup \gamma_{1}=\partial C$ for an incompressible, $\partial$-incompressible annulus $C \subset M$;

(ii) $\gamma_{0}$ is isotopic in $M$ to a curve $\gamma_{1} \subset \partial_{1} M$; or

(iii) $\gamma_{0}$ is isotopic in $M$ to a curve $\delta \subset F_{i}$ for some $i$.

Then $\exists$ an isotopy of $\gamma_{0}$ in $\partial_{0} M$ such that, after the isotopy, $\gamma_{0} \cap \partial V=\varnothing$.

ProOF. (i) Let $D$ be an annulus in the collection $\omega$, and consider $C \cap D$. Use $\partial$ incompressibility of $C$ to remove inessential $\operatorname{arcs}$ of $C \cap D$. Suppose $\alpha$ is an essential arc of $C \cap D$; then there must be other, parallel arcs of $C \cap \omega$ in $C$. Choose the component $E$ of $C \backslash \omega$ which contains $\alpha$ in its boundary and which lies in $V$. Let $\beta$ be the other arc of $C \cap \omega$ in $\partial E$. If $\alpha \subset\left(\partial f_{i}\right) \times I$, the fact that spanning arcs of $\left(\partial f_{k}\right) \times I(k \neq i)$ represent different classes of $H_{1}(M, \partial M)$ from $\alpha$ implies that $\beta \subset\left(\partial f_{i}\right) \times I$ as well. Thus $E$ may be pushed out of $V$ into $f_{k} \times I$. Finish the proof by induction.

(ii) Use the Generalized Dehn's Lemma [SW] to find an embedded annulus as in the statement of condition (i).

(iii) The isotopy can be extended from $F_{i}$ to $\partial_{1} M$ by noticing there is a "reflection" of $M$ in $F_{i}$. That is, if $i=1$, the map taking $f_{1} \times j \rightarrow f_{1} \times(1-j)$, $f_{2} \times j \rightarrow f_{3} \times(1-j)$, and $f_{3} \times j \rightarrow f_{2} \times(1-j)$ can be extended to $V$ as a homeomorphism. Q.E.D.

2. Construction of $N$. A component $\partial_{j} M$ of $\partial M$ can be seen in Figure 2 . Let $\eta_{j}: \partial_{j} M \rightarrow \partial_{j} M$ be the product of Dehn twists in the two bold curves shown in Figure 2. Notice that for any simple closed curve $\gamma$ in $\partial_{j} M$ with $\gamma \cap \partial \rho=\varnothing$ and $[\gamma] \neq 1 \in \pi_{1}\left(\partial_{j} M\right), \eta_{j}(\gamma) \cap \partial \rho \neq \varnothing$ and each arc of $\eta_{j}(\gamma) \backslash \partial \rho$ is essential in the component of $\partial_{j} M \backslash \partial \rho$ which contains it. (In general, any curves with these properties suffice for this construction.) Define $N$ to be two copies of $M$ glued along their boundaries by $\eta_{j}$. Pick one copy of $M \subset N$ to be referred to as $M$; the other will be $M^{\prime}$.

LEMMA 3. $N$ is irreducible and atoroidal. $\partial_{j} M$ is $x$-minimizing for $\left[\partial_{j} M\right] \in$ $H_{2}(N)$.

Proof. Since the $\partial_{j} M$ are incompressible and $M$ is irreducible, $N$ is irreducible. Consider a torus $T \subset N$ in general position with respect, to $\partial M$. Remove inessential curves of $T \cap \partial M$; then $T \cap \partial M$ consists of parallel essential curves on $T$. Now note that, if $D$ is an annular component of $T \backslash \partial M$ lying in $M$, either $D$ is $(\partial-)$ compressible or both adjacent annuli of $T \backslash \partial M$ are $(\partial-)$ compressible in $M^{\prime}$ (this uses Lemma 2 and the construction of $N$ ). Thus, either $T$ is compressible or $T \cap \partial M$ may be isotopically reduced until $T$ lies in $M$ or $M^{\prime}$ and bounds a solid torus.

If $H_{j}$ is incompressible and $x$-minimizing for $\left[\partial_{j} M\right]$ in $N$, an argument of Gabai (Lemma 3.6 of $[\mathbf{G}]$ ) shows that $H_{j}$ may be taken as disjoint from $\partial M$. Then $H_{j} \subset M$ or $M^{\prime}$, implying that $x\left(H_{j}\right)=x\left(\partial_{j} M\right)$. Q.E.D. 


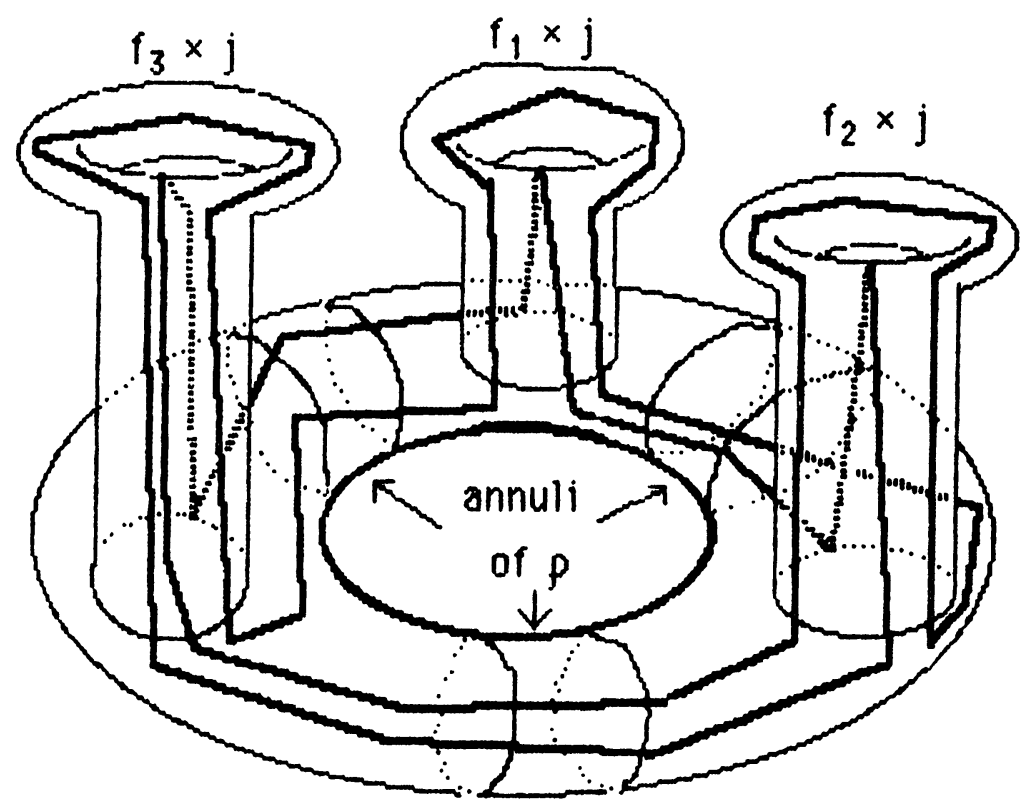

FIGURE 2

LEMMA 4. Let $H_{i}$ be a genus-2 surface in $N$ with $\left[H_{i}\right]=\left[F_{i}\right] \in H_{2}(N)$. Then $\exists$ an isotopy of $H_{i}$ in $N$ taking $H_{i}$ to $F_{i}$.

ProOF. For convenience, construct the cover $\Pi: \tilde{N} \rightarrow N$ using countably many copies of $M$ glued alternately using $\eta_{0}$ and $\eta_{1}$, indexing the copies of $M$ so that $\Pi\left(M_{0}\right)=M, \Pi\left(M_{ \pm 1}\right)=M^{\prime}, \Pi\left(M_{ \pm 2}\right)=M$, etc. Let $\tilde{F}_{i}$ be the lift of $F_{i}$ into $M_{0}$ and $\tilde{H}_{i}$ the corresponding lift of $H_{i}$. Let $k_{+}$be the largest index $n$ such that $M_{n} \cap \tilde{H}_{i} \neq \varnothing$; define $k_{-}$analogously. Induct on $k=k_{+}-k_{-} . k=0$ is Lemma 1 (iii).

Without loss of generality, assume that $n=k_{+}>0$. Let $Y=\tilde{H}_{i} \cap M_{n}$. Use the product structure on $\left(f_{j} \times I\right) \subset M_{n}$ to push $Y \cap\left(\left(f_{j} \times I\right) \subset M_{n}\right)$ into $M_{n-1}$. Similarly, push any $\partial$-parallel components of $Y$ remaining in $V \subset M_{n}$ into $M_{n-1}$. Let what is left of $Y$ in $M_{n}$ still be called $Y$.

Claim. $Y=\varnothing$.

If not, then $[Y]=0 \in H_{2}\left(M_{n}, \partial M_{n}\right)$ but each component of $Y$ is nontrivial in homology. This forces $Y$ to be pairs of essential annuli in $V \subset M_{n}$ with boundary components lying exclusively on $\partial_{0} M_{n}$ or $\partial_{1} M_{n}$ : say $\partial_{0} M_{n}$. Let $\tilde{H}_{i}^{\prime}$ be $\tilde{H}_{i}$ after surgery on $\tilde{H}_{i}$ along $\partial_{0} M_{n}$ and after discarding the resulting toral components of $M_{n}$ (see Figure 3).

$\tilde{H}_{i}^{\prime}$ has a smaller $k$ than $\tilde{H}_{i}$, so by induction $\tilde{H}_{i}^{\prime}$ is isotopic to $\tilde{F}_{i}$; in particular, the curves of $\partial Y$ are isotopic either to curves in $\partial_{1} M_{n-1}$ (if $n>1$ ) or in $\tilde{F}_{i}$ (if $n=1$ ). By Lemma 2 and the map $\eta_{0}, \partial Y$ would have to have nontrivial intersection with $\partial \rho \subset M_{n}$. This contradiction establishes the Claim and finishes the proof. Q.E.D.

THEOREM. There is a face of the $x$-unit sphere in $H_{2}(N)$ which is not spanned by a taut oriented branched surface. 


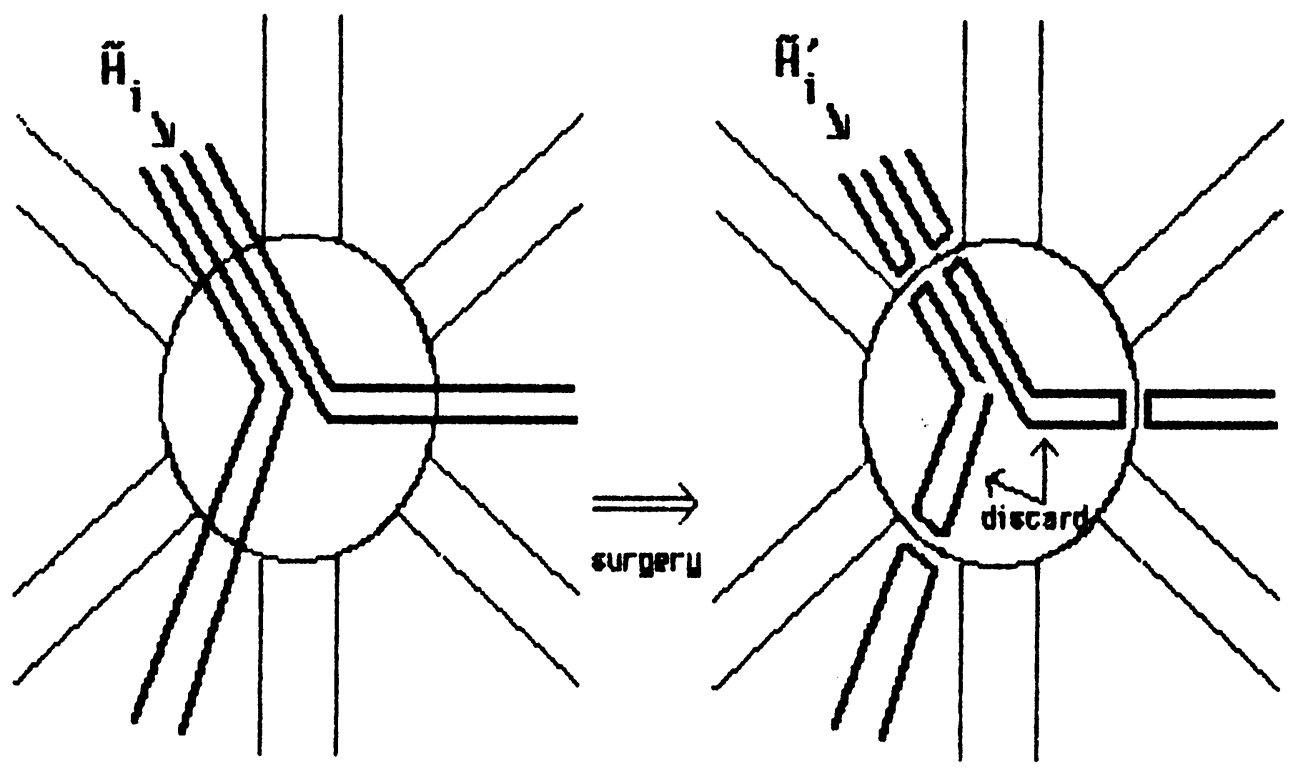

FIGURE 3

ProOF. Since $F_{1}, F_{2}, F_{3}$ and $F_{1}+F_{2}+F_{3}$ are all $x$-minimizing representatives of their respective classes in $H_{2}(N)$, their classes project to the same face $\varphi$ of the unit sphere. Let $\mathbf{B}$ be a branched surface spanning $\varphi$, and let $H_{i} \subset N$ be surfaces carried by $\mathbf{B}$ such that $\left[H_{i}\right]=\left[F_{i}\right] \in H_{2}(N)$ and each $H_{i}$ has genus 2 . Then the proof of Lemma 4 constructs an isotopy of $\partial_{0} M$ and $\partial_{1} M$ in $N$ which results in $H_{i} \subset M$. By Lemma $1, H_{1}+H_{2}+H_{3}$ contains a homologically trivial component $D$ in $V$. B carries $\sum H_{i}$, so it must carry $D$. Q.E.D.

\section{REFERENCES}

[G] D. Gabai, Foliations and the topology of 3-manifolds, J. Differential Geom. 18 (1983), 445-503.

[SW] A. Shapiro and H. Whitehead, A proof and extension of Dehn's lemma, Bull. Amer. Math. Soc. 64 (1958), 174-178.

Division of Physical and Biological Sciences, St. Edward's University, AUSTIN, TEXAS 78704 